Egyptian Journal of Aquatic Biology \& Fisheries

Zoology Department, Faculty of Science,

Ain Shams University, Cairo, Egypt.

ISSN $1110-6131$

Vol. 23(2): 517- 526 (2019)

www.ejabf.journals.ekb.eg

\title{
Factors Affecting Fish Blood Profile: A- Effect of Nutritional Treatments
}

\author{
Abdelhamid M. Abdelhamid ${ }^{1 *}$; Mohamed M. Refaey ${ }^{1}$; Mahmoud F. Salem ${ }^{2}$ and \\ Mostafa A. M. El-Kattan ${ }^{1}$ \\ 1- Animal Production Department, Faculty of Agriculture, Mansoura University, Egypt \\ 2- Aquaculture Research Unit, Sakha, Central Lab. of Aquaculture Research, Agricultural \\ Research Center, Cairo, Egypt \\ *Corresponding author: abdmohabd@ hotmail.com
}

\section{ARTICLE INFO}

Article History:

Received: May 9, 2019

Accepted: May 31, 2019

Online: June 2019

\section{Keywords:}

Fish

Haematology

Biochemistry

Nutrition

Diet type

Probiotics

\section{ABSTRACT}

Blood profile was studied as affected by different fish species, dietary crude protein, replacements, and additives, as well as diets' types, in laboratorial and field studies. It was clear that most studied treatments (food types, probiotic levels, levels of clover seed wastes, initial body weight, and Teen Barshomy wastes and levels) had been significantly affected most studied haematological and/or biochemical parameters. That means that fish blood constituents are not stable within known ranges, but widely varied according to various environmental conditions.

\section{INTRODUCTION}

Press and Evensen (1999) mentioned that species variation in the morphology of the immune system is to be expected, given the large number and diversity of species within the teleost fishes. Several external and internal factors can influence the activity of innate immune parameters. Temperature changes, handling and crowding stress can have suppressive effects on innate parameters, whereas several food additives and immunostimulants can enhance different innate factors. There is limited data available about the ontogenic development of the innate immunological system in fish (Magnadóttir, 2006). Moreover, Sahan and Duman (2008) found that haematocrit, leucocytes, monocytes, and neutrophils were increased in common carp fed beta glucan. Also, Aly et al. (2008) showed significant increase in haematocrit values in group of Nile tilapia fed the mixture of B. subtilis and L. acidophilus comparing with the control. However, the present study was designed to evaluate the effect of different nutritional treatments on some blood parameters.

\section{MATERIALS AND METHODS}

Random fish samples were taken for blood collected from the caudal peduncle by special syringe, adequate amount of whole blood was withdrawn in small plastic 
vials containing EDTA (ethylene diamine tetra acetic acid) as anticoagulant and used to obtain the blood plasma by centrifuge at $3500 \mathrm{rpm}$ for $15 \mathrm{~min}$. Blood plasma samples were used for determination of creatinine (Tietz, 1986), triglycerides (McGowan et al., 1983), total proteins (Tietz, 1990) and albumin (Wotton and Freeman, 1982) concentrations, as well as the activity of aspartate amino transferase (AST) and alanine amino transferase (ALT) using commercial test kits in a private lab. in Kafr El-Sheikh governorate, Egypt. Globulin level was calculated by subtracting albumin from total protein. The other samples of blood were used to determine the hematological parameters as concentration of hemoglobin $(\mathrm{Hb})$, total count of erythrocytes (RBCs), and total leukocytes (WBC S (Natt and Herrick, 1952) and hematocrit (Hct) using Auto Counter (Decie and Lewis, 2006) in the same lab. The other hematological parameters were mathematically calculated. Five fishes from each treatment in each case were chosen to withdraw blood samples to study the effects of different nutritional treatments on blood profile as followings:

1- Field study (in a private fish farm, Hag Aboulenin, in Metubus - Kafr El-Sheikh governorate during season 2017) of feeding Nile tilapia for 96 days on graded levels of a probiotic (PRO-LYNE ${ }^{\circledR}$ ) addition of floating and sinking diets.

2- In-door laboratorial study (Aquaculture Research Unit, Sakha, Central Lab. of Aquaculture Research, Agricultural Research Center, Ministry of Agriculture, Cairo, Egypt) on feeding Mono-sex Nile tilapia diets containing graded levels of ground wastes of sieving the Egyptian clover seeds instead of the dietary soybean meal on basis of crude protein content, for 57 days during 2017.

3- Field study in Mahmud Hussein Hatchery, Tolombat-7, Al-Reiad, Kafr El-Sheikh governorate during season 2017 to study the effect of dietary crude protein levels $(27,32$, and $38 \%)$ on common carp for 6 months during 2018.

4- To study the effect of feeding all-males mono-sex Nile tilapia for 75 days on diets containing 25 and $50 \%$ fig skins and leaves instead of the dietary corn.

All obtained data were analyzed according to statistical analysis system software (SAS, 2006) for windows. Duncan's multiple range tests (Duncan, 1955) were used to compare between the parameters of the different nutritional groups. The differences were significant at 0.05 levels.

\section{RESULTS}

A study was conducted over 96 days in order to comprise between floating and sinking diets supplemented with and/or without a probiotic and their effects on growth performance chemical composition and food utilization of Nile tilapia (Oreochromis niloticus). The two studied factors were the diet type (floating and sinking diets) and probiotic (PRO-LYNE ${ }^{\circledR}$, at $0 \%, 1 \%$ and $2 \%$ of the diet). Food type (floating or sinking diets) significantly affected blood values of Hct, mean corpuscular haemoglobin concentration (MCHC), platelets and WBCs; whereas the probiotic level affected all tested haematological parameters (Table 1), except RBCs, mean corpuscular volume (MCV), and mean corpuscular haemoglobin (MCH). Meanwhile, the only significant interaction (food type $\times$ probiotic level) was calculated for Hct and MCH. Concerning the biochemical parameters (Table 2), food type significantly affected on urea, triglycerides (TG), and low density lipoprotein (LDL) only. The probiotic level did not affect the measured biochemical parameters. Yet, the interaction was significant for AST, ALT, and high density lipoprotein (HDL). 
Table 1: Impact of diet type and probiotic on haematological parameters of the Nile tilapia

\begin{tabular}{|c|c|c|c|c|c|c|c|c|}
\hline & $\begin{array}{c}\mathbf{H b} \\
(\mathrm{g} / \mathrm{dl})\end{array}$ & $\begin{array}{l}\text { Hct } \\
(\%)\end{array}$ & $\begin{array}{c}\text { RBCs } \\
(\mathbf{X ~ 1 0} / \mu \mathrm{l})\end{array}$ & $\begin{array}{c}\text { MCV } \\
\text { (fl) }\end{array}$ & $\begin{array}{c}\text { MCH } \\
\text { (pg) }\end{array}$ & $\begin{array}{c}\text { MCHC } \\
(\%)\end{array}$ & $\begin{array}{l}\text { Platelets } \\
\left(\mathrm{X} 10^{3} / \mu \mathrm{I}\right)\end{array}$ & $\begin{array}{c}\text { WBCs } \\
\left(\mathrm{X} \mathrm{10}^{3} / \mu \mathrm{l}\right)\end{array}$ \\
\hline \multicolumn{9}{|l|}{ Food types } \\
\hline \multirow{2}{*}{ Floating } & 11.23 & $32.42^{\mathrm{a}}$ & 2224 & 133.6 & 20.27 & $28.73^{\mathrm{b}}$ & $125000^{\mathrm{a}}$ & $69067^{\mathrm{a}}$ \\
\hline & \pm 0.26 & \pm 1.30 & \pm 155.0 & \pm 0.90 & \pm 0.44 & \pm 0.66 & \pm 8409 & \pm 3173 \\
\hline \multirow[t]{2}{*}{ Sinking } & 11.03 & $28.36^{\mathbf{b}}$ & 2000 & 135.0 & 20.40 & $30.33^{\mathrm{a}}$ & $95000^{\mathbf{b}}$ & $56367^{b}$ \\
\hline & \pm 0.20 & \pm 0.88 & \pm 36.00 & \pm 0.81 & \pm 0.49 & \pm 0.36 & \pm 7528 & \pm 2315 \\
\hline \multicolumn{9}{|l|}{ Additive (\%) } \\
\hline \multirow[t]{2}{*}{ 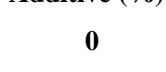 } & $11.60^{\mathrm{a}}$ & $31.44^{\mathrm{a}}$ & 2075 & 135.6 & 20.30 & $28.20^{\mathbf{b}}$ & $103000^{\text {ab }}$ & $71850^{\mathrm{a}}$ \\
\hline & \pm 0.28 & \pm 0.93 & \pm 50.69 & \pm 0.99 & \pm 0.65 & \pm 0.92 & \pm 12806 & \pm 4224 \\
\hline \multirow{2}{*}{1} & $10.70^{\mathbf{b}}$ & $27.92^{\mathbf{b}}$ & 2235 & 133.1 & 20.00 & $29.80^{\mathbf{a b}}$ & $127000^{\mathrm{a}}$ & $63700^{\mathbf{b}}$ \\
\hline & \pm 0.24 & \pm 1.14 & \pm 229.1 & \pm 1.05 & \pm 0.56 & \pm 0.39 & \pm 10651 & \pm 2336 \\
\hline \multirow{2}{*}{2} & $11.10^{\mathbf{a b}}$ & $31.81^{\mathrm{a}}$ & 2027 & 134.2 & 20.70 & $30.60^{\mathrm{a}}$ & $100000^{\mathrm{b}}$ & $52600^{c}$ \\
\hline & \pm 0.28 & \pm 1.99 & \pm 74.58 & \pm 1.07 & \pm 0.50 & \pm 0.45 & \pm 6791 & \pm 2322 \\
\hline \multicolumn{9}{|l|}{ Interactions } \\
\hline \multirow{2}{*}{ Floating $* 0$} & 11.70 & 31.12 & 2100 & 134.4 & 19.00 & 26.60 & 130000 & 81700 \\
\hline & \pm 0.46 & \pm 1.45 & \pm 74.16 & \pm 1.72 & \pm 0.63 & \pm 1.54 & \pm 12450 & \pm 5578 \\
\hline \multirow{2}{*}{ Floating $* 1$} & 10.70 & 28.94 & 2443 & 131.2 & 20.00 & 29.40 & 146000 & 67400 \\
\hline & \pm 0.37 & \pm 2.15 & \pm 460.4 & \pm 1.07 & \pm 0.63 & \pm 0.51 & \pm 14265 & \pm 1187 \\
\hline \multirow{2}{*}{ Floating $* 2$} & 11.30 & 37.19 & 2129 & 135.2 & 21.80 & 30.20 & 99000 & 58100 \\
\hline & \pm 0.46 & \pm 1.39 & \pm 129.0 & \pm \pm 1.46 & \pm 0.49 & \pm 0.37 & \pm 9925 & \pm 1592 \\
\hline \multirow[t]{2}{*}{ Sinking*0 } & 11.50 & 31.76 & 2049 & 136.8 & 21.60 & 29.80 & 76000 & 62000 \\
\hline & \pm 0.35 & \pm 1.32 & \pm 75.75 & \pm 0.86 & \pm 0.81 & \pm 0.37 & \pm 14782 & \pm 806 \\
\hline \multirow[t]{2}{*}{ Sinking*1 } & 10.70 & 26.90 & 2026 & 135.0 & 20.00 & 30.20 & 108000 & 60000 \\
\hline & \pm 0.34 & \pm 0.84 & \pm 50.18 & \pm 1.41 & \pm 1.00 & \pm 0.58 & \pm 11247 & \pm 4037 \\
\hline \multirow[t]{2}{*}{ Sinking*2 } & 10.90 & 26.43 & 1924 & 133.2 & 19.60 & 31.00 & 101000 & 47100 \\
\hline & \pm 0.33 & \pm 1.18 & \pm 56.02 & \pm 1.59 & \pm 0.51 & \pm 0.84 & \pm 10416 & \pm 2571 \\
\hline P value & 0.8783 & 0.0016 & 0.6665 & 0.1135 & 0.0085 & 0.2517 & 0.0847 & 0.1511 \\
\hline
\end{tabular}

a-c: Mean superscripted with different letters in the same column and group differ significantly at $\mathrm{P} \leq 0.05, \mathrm{Hb}$ : haemoglobin, Hct: haematocrit, RBCs: red blood cells, MCV: mean corpuscular volume, MCH: mean corpuscular haemoglobin, MCHC: mean corpuscular haemoglobin concentration, WBCs: white blood cells.

Table 2: Impact of diet type and probiotic on biochemical parameters of the Nile tilapia

\begin{tabular}{|c|c|c|c|c|c|c|c|c|c|c|c|}
\hline & $\begin{array}{l}\text { AST } \\
(\mathrm{u} / \mathbf{l})\end{array}$ & $\begin{array}{c}\text { ALT } \\
(\mathbf{u} / \mathbf{l})\end{array}$ & $\begin{array}{c}\mathbf{A L} \\
\text { (g/dl) }\end{array}$ & $\begin{array}{c}\text { GL } \\
(\mathbf{g} / \mathbf{d l})\end{array}$ & $\begin{array}{c}\text { TP } \\
(\mathbf{g} / \mathbf{d l})\end{array}$ & $\begin{array}{c}\text { Creatinine } \\
(\mathrm{mg} / \mathrm{dl})\end{array}$ & $\begin{array}{l}\text { Urea } \\
(\mathrm{mg} / \mathrm{dl})\end{array}$ & $\begin{array}{c}\text { TCH } \\
(\mathrm{mg} / \mathrm{dl})\end{array}$ & $\begin{array}{c}\text { TG } \\
(\mathbf{m g} / \mathrm{dl})\end{array}$ & $\begin{array}{c}\text { HDL } \\
(\mathrm{mg} / \mathrm{dl})\end{array}$ & $\begin{array}{c}\text { LDL } \\
(\mathrm{mg} / \mathrm{dl})\end{array}$ \\
\hline \multicolumn{12}{|l|}{ Food types } \\
\hline \multirow[t]{2}{*}{ Floating } & 174.5 & 51.27 & 5.12 & 1.23 & 3.47 & 0.30 & $12.27^{\mathbf{b}}$ & 284.8 & $338.2^{\mathrm{a}}$ & 142.5 & $80.27^{\mathrm{a}}$ \\
\hline & \pm 11.40 & \pm 3.35 & \pm 0.10 & \pm 0.03 & \pm 0.11 & \pm 0.01 & \pm 0.91 & \pm 7.84 & \pm 16.23 & \pm 4.13 & \pm 5.47 \\
\hline \multirow[t]{2}{*}{ Sinking } & 189.2 & 52.07 & 4.84 & 1.18 & 3.39 & 0.31 & $16.27^{\mathrm{a}}$ & 272.3 & $287.2^{b}$ & 150.3 & $64.73^{b}$ \\
\hline & \pm 9.70 & \pm 3.97 & \pm 0.10 & \pm 0.02 & \pm 0.11 & \pm 0.01 & \pm 0.85 & \pm 6.97 & \pm 18.04 & \pm 6.15 & \pm 5.01 \\
\hline \multicolumn{12}{|l|}{ Additive (\%) } \\
\hline \multirow[t]{2}{*}{$\mathbf{0}$} & 189.8 & 46.70 & 4.94 & 1.16 & 3.42 & 0.31 & 14.30 & 283.1 & 298.6 & 149.3 & 75.70 \\
\hline & \pm 11.42 & \pm 3.70 & \pm 0.16 & \pm 0.04 & \pm 0.17 & \pm 0.01 & \pm 1.10 & \pm 9.97 & \pm 26.72 & \pm 8.31 & \pm 7.54 \\
\hline \multirow[t]{2}{*}{1} & 186.4 & 52.40 & 5.10 & 1.20 & 3.50 & 0.30 & 13.40 & 280.7 & 332.1 & 150.4 & 69.60 \\
\hline & \pm 11.35 & \pm 4.24 & \pm 0.13 & \pm 0.04 & \pm 0.12 & \pm 0.01 & \pm 1.47 & \pm 12.04 & \pm 22.27 & \pm 5.99 & \pm 6.92 \\
\hline \multirow[t]{2}{*}{2} & 169.4 & 55.90 & 4.91 & 1.25 & 3.36 & 0.32 & 15.10 & 271.9 & 307.4 & 139.4 & 72.20 \\
\hline & \pm 15.97 & \pm 5.15 & \pm 0.09 & \pm 0.03 & \pm 0.10 & \pm 0.02 & \pm 1.21 & \pm 4.23 & \pm 17.72 & \pm 4.47 & \pm 6.45 \\
\hline \multicolumn{12}{|l|}{ Interactions } \\
\hline \multirow[t]{2}{*}{ Floating *0 } & 187.0 & 51.00 & 5.20 & 1.14 & 3.64 & 0.33 & 12.40 & 278.6 & 338.6 & 129.6 & 84.60 \\
\hline & \pm 16.82 & \pm 7.14 & \pm 0.26 & \pm 0.07 & \pm 0.25 & \pm 0.02 & \pm 1.29 & \pm 18.12 & \pm 34.55 & \pm 8.62 & \pm 13.33 \\
\hline \multirow[t]{2}{*}{ Floating $* 1$} & 199.6 & 58.00 & 5.22 & 1.24 & 3.54 & 0.29 & 10.20 & 298.4 & 378.4 & 149.0 & 84.60 \\
\hline & \pm 12.83 & \pm 5.39 & \pm 0.14 & \pm 0.05 & \pm 0.14 & \pm 0.01 & \pm 1.66 & \pm 14.60 & \pm 13.84 & \pm 6.19 & \pm 6.42 \\
\hline \multirow[t]{2}{*}{ Floating $* 2$} & 137.0 & 44.80 & 4.94 & 1.30 & 3.22 & 0.30 & 14.20 & 277.4 & 297.6 & 148.8 & 71.60 \\
\hline & \pm 19.24 & \pm 4.00 & \pm 0.11 & \pm 0.04 & \pm 0.11 & \pm 0.02 & \pm 1.53 & \pm 5.91 & \pm 23.75 & \pm 2.08 & \pm 8.18 \\
\hline \multirow[t]{2}{*}{ Sinking*0 } & 192.6 & 42.40 & 4.67 & 1.18 & 3.20 & 0.29 & 16.20 & 287.6 & 258.6 & 169.0 & 66.80 \\
\hline & \pm 17.33 & \pm 1.12 & \pm 0.13 & \pm 0.04 & \pm 0.21 & \pm 0.02 & \pm 1.39 & \pm 10.43 & \pm 34.91 & \pm 6.53 & \pm 6.22 \\
\hline \multirow[t]{2}{*}{ Sinking*1 } & 173.2 & 46.80 & 4.99 & 1.16 & 3.46 & 0.30 & 16.60 & 263.0 & 285.8 & 151.8 & 54.60 \\
\hline & \pm 18.12 & \pm 6.03 & \pm 0.22 & \pm 0.05 & \pm 0.21 & \pm 0.01 & \pm 1.36 & \pm 16.79 & \pm 31.12 & \pm 11.05 & \pm 7.87 \\
\hline \multirow[t]{2}{*}{ Sinking*2 } & 201.8 & 67.00 & 4.87 & 1.20 & 3.50 & 0.35 & 16.00 & 266.0 & 317.2 & 130.0 & 72.80 \\
\hline & \pm 15.92 & \pm 6.44 & \pm 0.14 & \pm 0.03 & \pm 0.15 & \pm 0.04 & \pm 1.95 & \pm 5.50 & \pm 28.30 & \pm 6.44 & \pm 10.95 \\
\hline Pvalue & 0.0374 & 0.0082 & 0.4223 & 0.3154 & 0.1735 & 0.1500 & 0.3450 & 0.2462 & 0.1224 & 0.0021 & 0.2511 \\
\hline
\end{tabular}

a-b: Mean superscripted with different letters in the same column and group differ significantly at P $\leq 0.05$, AST: aspartate aminotransferase, ALT: alanine aminotransferase, GL: globulin, TP: total protein, TCH: total cholesterol, TG: triglycerides, HDL: high density lipoprotein, LDL: low density lipoprotein.

A feeding experiment for 57 days was conducted on Nile tilapia to evaluate the effects of dietary inclusion of graded levels $(0,25,50,75$, and 100\%) of sieving 
wastes meal of Egyptian clover seeds instead of soybean meal based on crude protein content. The replacement level significantly affected all tested haematological parameters, except MCH (Table 3). It also affects albumin (AL), urea, total cholesterol (TCH), and HDL (Table 4).

Table 3: Impact of dietary inclusion of graded levels of sieving wastes meal of Egyptian clover seed on haematological parameters of the Nile tilapia

\begin{tabular}{|c|c|c|c|c|c|c|c|c|}
\hline Treatments & $\begin{array}{c}\mathbf{H b} \\
(\mathrm{g} / \mathrm{dl})\end{array}$ & $\begin{array}{l}\text { Het } \\
(\%)\end{array}$ & $\begin{array}{c}\text { RBCs } \\
(\mathrm{X} \mathrm{10} / \mu \mathrm{l})\end{array}$ & $\begin{array}{c}\text { MCV } \\
\text { (fl) }\end{array}$ & $\begin{array}{c}\text { MCH } \\
\text { (pg) }\end{array}$ & $\begin{array}{c}\text { MCHC } \\
(\%)\end{array}$ & $\begin{array}{c}\text { Platelets } \\
(\mathrm{X} \mathrm{10} / \mu \mathrm{l})\end{array}$ & $\begin{array}{c}\text { WBCs } \\
\left(\mathrm{X} \mathrm{10}^{3} / \mu \mathrm{l}\right)\end{array}$ \\
\hline \multirow[t]{2}{*}{$0 \%$} & $11.84^{\mathrm{a}}$ & $26.69^{a}$ & $2130^{\mathrm{a}}$ & $124.2^{\mathrm{a}}$ & 55.20 & $43.64^{b}$ & $121800^{a}$ & $84600^{\mathrm{a}}$ \\
\hline & \pm 0.52 & \pm 1.12 & \pm 124.5 & \pm 3.06 & \pm 1.17 & \pm 0.36 & & \pm 5250 \\
\hline \multirow[t]{2}{*}{$50 \%$} & $9.12^{b}$ & $21.22^{\mathrm{b}}$ & $1674^{\mathbf{b}}$ & $127.4^{\mathrm{a}}$ & 53.38 & $41.60^{c}$ & $122800^{\mathbf{a}}$ & $64600^{b}$ \\
\hline & \pm 0.44 & \pm 1.23 & \pm 76.00 & \pm 2.50 & \pm 1.37 & \pm 0.51 & \pm 1594 & \pm 5240 \\
\hline \multirow[t]{2}{*}{$75 \%$} & $12.74^{\mathrm{a}}$ & $30.83^{\mathrm{a}}$ & $2472^{\mathrm{a}}$ & $128.0^{\mathrm{a}}$ & 52.32 & $42.26^{\text {bc }}$ & $130400^{\mathbf{a}}$ & $93400^{\mathrm{a}}$ \\
\hline & \pm 0.56 & \pm 1.77 & \pm 159.9 & \pm 0.95 & \pm 0.66 & \pm 0.62 & \pm 3400 & \pm 6735 \\
\hline \multirow[t]{2}{*}{$100 \%$} & $8.02^{b}$ & $18.49^{b}$ & $1675^{b}$ & $115.4^{\mathbf{b}}$ & 52.20 & $46.24^{\mathrm{a}}$ & $98800^{b}$ & $46600^{c}$ \\
\hline & \pm 0.55 & \pm 2.08 & \pm 149.3 & \pm 3.88 & \pm 2.08 & \pm 0.41 & \pm 2557 & \pm 4468 \\
\hline
\end{tabular}

a-c: Mean superscripted with different letters in the same column and group differ significantly at $\mathrm{P} \leq 0.05$, Hb: haemoglobin, Hct: haematocrit, RBCs: red blood cells, MCV: mean corpuscular volume, $\mathrm{MCH}$ : mean corpuscular haemoglobin, MCHC: mean corpuscular haemoglobin concentration, WBCs: white blood cells. .

Table 4: Impact of dietary inclusion of graded levels of sieving wastes meal of Egyptian clover seed on biochemical parameters of the Nile tilapia

\begin{tabular}{cccccccccccc}
\hline Treatments & $\begin{array}{c}\mathbf{A S T} \\
(\mathbf{u} / \mathbf{l})\end{array}$ & $\begin{array}{c}\mathbf{A L T} \\
(\mathbf{u} / \mathbf{l})\end{array}$ & $\begin{array}{c}\mathbf{A L} \\
(\mathbf{g} / \mathbf{d l})\end{array}$ & $\begin{array}{c}\mathbf{G L} \\
(\mathbf{g} / \mathbf{d l})\end{array}$ & $\begin{array}{c}\text { TP } \\
(\mathbf{g} / \mathbf{d l})\end{array}$ & $\begin{array}{c}\text { Creatinine } \\
(\mathbf{m g} / \mathbf{d l})\end{array}$ & $\begin{array}{c}\text { Urea } \\
(\mathbf{m g} / \mathbf{d l})\end{array}$ & $\begin{array}{c}\text { TCH } \\
(\mathbf{m g} / \mathbf{d l})\end{array}$ & $\begin{array}{c}\text { TG } \\
(\mathbf{m g} / \mathbf{d l})\end{array}$ & $\begin{array}{c}\text { HDL } \\
(\mathbf{m g} / \mathbf{d l})\end{array}$ & $\begin{array}{c}\text { LDL } \\
(\mathbf{m g} / \mathbf{d l})\end{array}$ \\
\hline $\mathbf{0 \%}$ & 314.8 & 148.0 & 4.22 & $1.33^{\mathbf{a}}$ & 2.68 & 0.12 & $13.80^{\mathbf{a}}$ & $150.80^{\mathbf{a b}}$ & 146.80 & $65.80^{\mathbf{a}}$ & 55.40 \\
& \pm 1.46 & \pm 7.62 & \pm 0.21 & \pm 0.03 & \pm 0.22 & \pm 0.00 & \pm 1.69 & \pm 3.07 & \pm 14.58 & \pm 1.66 & \pm 3.49 \\
$\mathbf{5 0 \%}$ & 324.0 & 126.4 & 4.33 & $1.29^{\mathbf{a b}}$ & 3.00 & 0.12 & $11.00^{\mathbf{a b}}$ & $146.40^{\mathbf{a b}}$ & 148.20 & $62.60^{\mathbf{b}}$ & 53.80 \\
& \pm 7.04 & \pm 6.01 & \pm 0.17 & \pm 0.03 & \pm 0.20 & \pm 0.00 & \pm 1.30 & \pm 3.17 & \pm 2.01 & \pm 0.81 & \pm 3.88 \\
$\mathbf{7 5 \%}$ & 311.8 & 134.8 & 4.40 & $1.27^{\mathbf{a b}}$ & 2.82 & 0.12 & $12.80^{\mathbf{a}}$ & $155.60^{\mathbf{a}}$ & 153.60 & $58.60^{\mathbf{c}}$ & 65.80 \\
& \pm 3.29 & \pm 11.02 & \pm 0.19 & \pm 0.03 & \pm 0.29 & \pm 0.00 & \pm 1.16 & \pm 4.35 & \pm 3.04 & \pm 0.51 & \pm 4.37 \\
$\mathbf{1 0 0 \%}$ & 311.2 & 124.2 & 4.04 & $1.23^{\mathbf{b}}$ & 2.62 & 0.11 & $8.80^{\mathbf{b}}$ & $142.40^{\mathbf{b}}$ & 145.20 & $58.80^{\mathbf{c}}$ & 54.20 \\
& \pm 0.73 & \pm 16.07 & \pm 0.14 & \pm 0.01 & \pm 0.12 & \pm 0.00 & \pm 0.66 & \pm 4.99 & \pm 2.27 & \pm 0.58 & \pm 4.84 \\
\hline
\end{tabular}

a-c: Means superscripted with different letters in the same column differ significantly at $\mathrm{P} \leq 0.05, \mathrm{AST}$ : aspartate aminotransferase, ALT: alanine aminotransferase, GL: globulin, TP: total protein, TCH: total cholesterol, TG: triglycerides, HDL: high density lipoprotein, LDL: low density lipoprotein.

A Field study in Mahmud Hussein Hatchery, Tolombat-7, Al-Reiad, Kafr ElSheikh governorate was conducted to study the effect of dietary crude protein levels (27, 32, and 38\%) on common carp for 6 months. The 32\%-CP-diet reflected the highest $(\mathrm{P} \leq 0.05)$ platelets' count (Table 5); yet, increasing dietary $\mathrm{CP} \%$ led to gradual increase $(\mathrm{P} \leq 0.05)$ in ALT activity, creatinine concentration, but gradual decreased $(\mathrm{P} \leq 0.05)$ triglycerides level (Table 6).

Table 5: Impact of dietary crude protein levels on haematological parameters of common carp

\begin{tabular}{|c|c|c|c|c|c|c|c|c|}
\hline Treat. & $\begin{array}{c}\text { Hb } \\
(\mathrm{g} / \mathrm{dl})\end{array}$ & $\begin{array}{l}\text { Het } \\
(\%)\end{array}$ & $\begin{array}{c}\text { RBCs } \\
(\mathrm{X} \mathrm{10} \% \mu \mathrm{l})\end{array}$ & $\begin{array}{l}\text { MCV } \\
\text { (fl) }\end{array}$ & $\begin{array}{c}\text { MCH } \\
\text { (pg) }\end{array}$ & $\begin{array}{c}\text { MCHC } \\
(\%)\end{array}$ & $\begin{array}{c}\text { Platelets } \\
(\mathrm{X} \mathrm{10} / \mu \mathrm{l})\end{array}$ & $\begin{array}{c}\text { WBCs } \\
(\mathrm{X} \mathrm{10} / \mu \mathrm{l})\end{array}$ \\
\hline \multirow{2}{*}{$27 \%$} & 14.22 & 22.84 & 1960 & 114.8 & 71.62 & 65.78 & $137600^{b}$ & 183400 \\
\hline & \pm 0.51 & \pm 1.30 & \pm 102.1 & \pm 4.02 & \pm 2.11 & \pm 3.46 & \pm 2379 & \pm 7852 \\
\hline \multirow{2}{*}{$32 \%$} & 13.62 & 24.07 & 2018 & 111.4 & 66.04 & 59.24 & $184000^{\mathrm{a}}$ & 197200 \\
\hline & \pm 0.84 & \pm 3.51 & \pm 172.6 & \pm 6.47 & \pm 2.03 & \pm 4.85 & \pm 13817 & \pm 11719 \\
\hline \multirow{2}{*}{$38 \%$} & 13.62 & 22.98 & 1950 & 113.8 & 67.44 & 60.10 & $151600^{\mathbf{a b}}$ & 180200 \\
\hline & \pm 0.17 & \pm 0.76 & \pm 42.78 & \pm 1.66 & \pm 1.04 & \pm 1.45 & \pm 13974 & \pm 5014 \\
\hline
\end{tabular}

a-b: Mean superscripted with different letters in the same column and group differ significantly at $\mathrm{P} \leq 0.05, \mathrm{Hb}$ : haemoglobin, Hct: haematocrit, RBCs: red blood cells, MCV: mean corpuscular volume, $\mathrm{MCH}$ : mean corpuscular haemoglobin, MCHC: mean corpuscular haemoglobin concentration, WBCs: white blood cells. . 
Table 6: Impact of dietary crude protein levels on biochemical parameters of the common carp

\begin{tabular}{cccccccccccc}
\hline Treat. & $\begin{array}{c}\mathbf{A S T} \\
(\mathbf{u} / \mathbf{l})\end{array}$ & $\begin{array}{c}\mathbf{A L T} \\
(\mathbf{u} / \mathbf{l})\end{array}$ & $\begin{array}{c}\mathbf{A L} \\
(\mathbf{g} / \mathbf{d l})\end{array}$ & $\begin{array}{c}\mathbf{G L} \\
(\mathbf{g} / \mathbf{d l})\end{array}$ & $\begin{array}{c}\text { TP } \\
(\mathbf{g} / \mathbf{d l})\end{array}$ & $\begin{array}{c}\text { Creatinine } \\
(\mathbf{m g} / \mathbf{d l})\end{array}$ & $\begin{array}{c}\text { Urea } \\
(\mathbf{m g} / \mathbf{d l})\end{array}$ & $\begin{array}{c}\text { TCH } \\
(\mathbf{m g} / \mathbf{d l})\end{array}$ & $\begin{array}{c}\text { TG } \\
(\mathbf{m g} / \mathbf{d l})\end{array}$ & $\begin{array}{c}\text { HDL } \\
(\mathbf{m g} / \mathbf{d l})\end{array}$ & $\begin{array}{c}\mathbf{L D L} \\
(\mathbf{m g} / \mathbf{d l})\end{array}$ \\
\hline $\mathbf{2 7 \%}$ & 73.80 & $19.40^{\mathbf{b}}$ & 3.58 & 0.80 & 2.46 & $0.25^{\mathbf{a b}}$ & 12.62 & 131.60 & $230.2^{\mathbf{a}}$ & 41.80 & 43.60 \\
& \pm 11.80 & \pm 0.87 & \pm 0.16 & \pm 0.03 & \pm 0.18 & \pm 0.01 & \pm 0.23 & \pm 2.09 & \pm 20.45 & \pm 0.80 & \pm 4.79 \\
$\mathbf{3 2 \%}$ & 83.60 & $23.80^{\mathbf{b}}$ & 3.52 & 0.82 & 2.44 & $0.23^{\mathbf{b}}$ & 12.60 & 139.40 & $186.8^{\mathbf{a b}}$ & 45.40 & 56.40 \\
& \pm 5.19 & \pm 1.62 & \pm 0.22 & \pm 0.04 & \pm 0.22 & \pm 0.01 & \pm 0.24 & \pm 9.50 & \pm 9.17 & \pm 1.96 & \pm 8.31 \\
$\mathbf{3 8 \%}$ & 98.40 & $30.40^{\mathbf{a}}$ & 3.37 & 0.84 & 2.22 & $0.27^{\mathbf{a}}$ & 13.00 & 133.80 & $176.4^{\mathbf{b}}$ & 50.80 & 47.40 \\
& \pm 12.11 & \pm 1.75 & \pm 0.11 & \pm 0.04 & \pm 0.15 & \pm 0.01 & \pm 0.45 & \pm 3.28 & \pm 10.71 & \pm 4.49 & \pm 2.73 \\
\hline
\end{tabular}

a-b: Means superscripted with different letters in the same column differ significantly at $\mathrm{P} \leq 0.05$, AST: aspartate aminotransferase, ALT: alanine aminotransferase, GL: globulin, TP: total protein, TCH: total cholesterol, TG: triglycerides, HDL: high density lipoprotein, LDL: low density lipoprotein.

A laboratory study was conducted to evaluate the effects of replacing 25 and $50 \%$ of the diet's corn by meals of fruit skin and leaves of Teen Barshomy (Teen Shoky) on Nile tilapia for 75 days. The replacement type (pee / waste) significantly affected WBCs only; whereas, the replacement level affected significantly all tested haematological parameters, except $\mathrm{MCV}, \mathrm{MCH}$, and MCHC. The interaction (replacement type $\times$ replacement level) was significant only for platelets (Table 7 ). The replacement type significantly affected TCH, HDL, and LDL. Moreover, the replacement level significantly affected total protein (TP), albumin (AL), globulin $(G L)$ and LDL (Table 8). The interaction (type $\times$ level of replacement) was significant only for $\mathrm{TCH}$.

Table 7: Impact of dietary inclusion of of fruit skin and leaves of Teen Barshomy on haematological parameters of the Nile tilapia

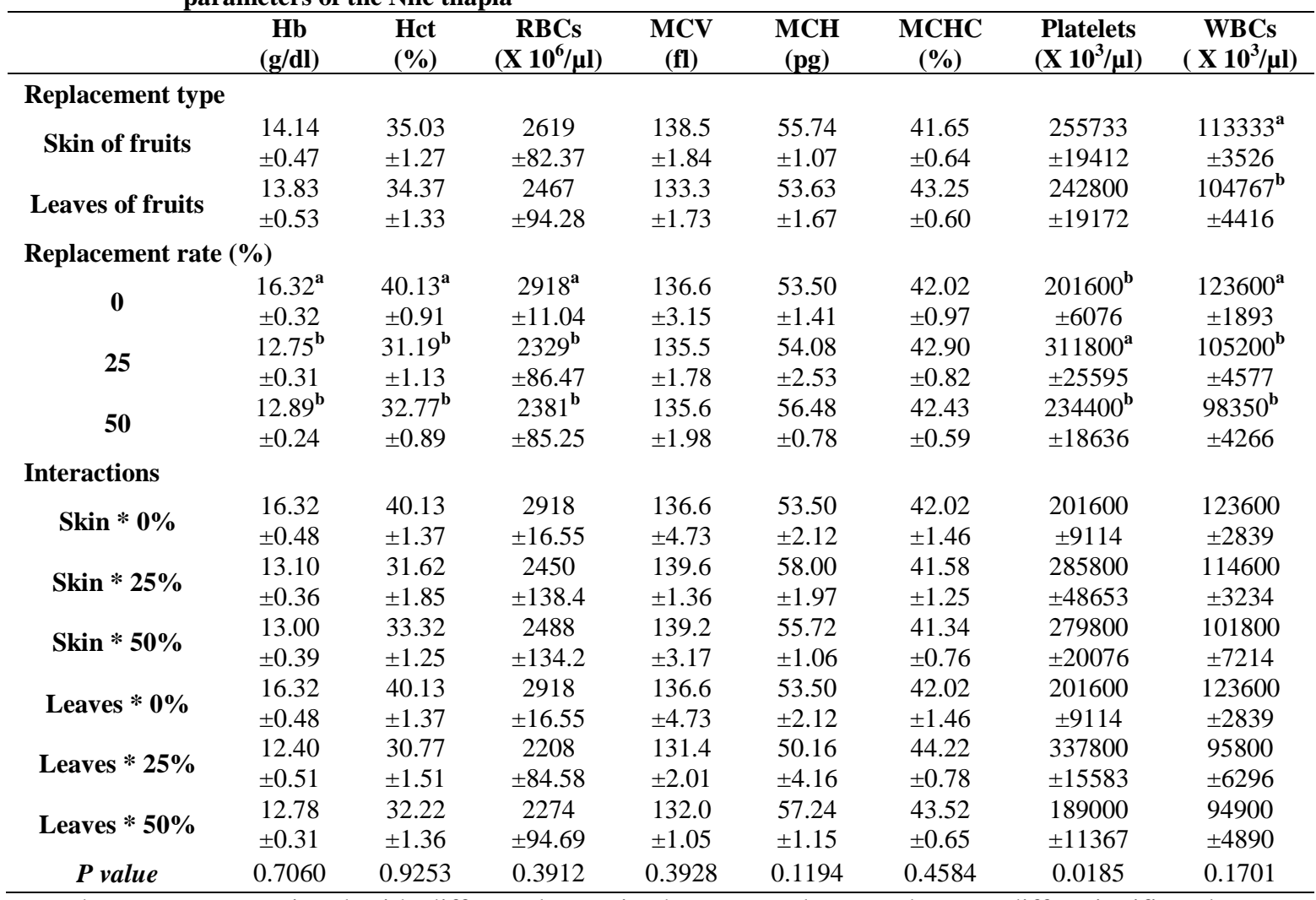

a-b: Mean superscripted with different letters in the same column and group differ significantly at $\mathrm{P} \leq 0.05$, Hb: haemoglobin, Hct: haematocrit, RBCs: red blood cells, MCV: mean corpuscular volume, MCH: mean corpuscular haemoglobin, MCHC: mean corpuscular haemoglobin concentration, WBCs: white blood cells. . 
Table 8: Impact of dietary inclusion of of fruit skin and leaves of Teen Barshomy on biochemical parameters of the Nile tilapia

\begin{tabular}{|c|c|c|c|c|c|c|c|c|c|c|c|}
\hline & $\begin{array}{l}\text { AST } \\
(\mathbf{u} / \mathbf{l})\end{array}$ & $\begin{array}{r}\text { ALT } \\
(\mathbf{u} / \mathbf{l})\end{array}$ & $\begin{array}{c}\mathbf{A L} \\
(\mathrm{g} / \mathrm{dl})\end{array}$ & $\begin{array}{c}\text { GL } \\
(\mathrm{g} / \mathrm{dl})\end{array}$ & $\begin{array}{c}\mathbf{T P} \\
(\mathrm{g} / \mathrm{dl})\end{array}$ & $\begin{array}{c}\text { Creatinine } \\
(\mathrm{mg} / \mathrm{dl})\end{array}$ & $\begin{array}{c}\text { Urea } \\
(\mathrm{mg} / \mathrm{dl})\end{array}$ & $\begin{array}{c}\text { TCH } \\
(\mathbf{m g} / \mathrm{dl})\end{array}$ & $\begin{array}{c}\text { TG } \\
(\mathbf{m g} / \mathrm{dl})\end{array}$ & $\begin{array}{c}\text { HDL } \\
(\mathrm{mg} / \mathrm{dl})\end{array}$ & $\begin{array}{c}\text { LDL } \\
(\mathbf{m g} / \mathbf{d l})\end{array}$ \\
\hline \multicolumn{12}{|c|}{ Replacement type } \\
\hline Skin of fruits & $\begin{array}{c}187.1 \\
\pm 23.95\end{array}$ & $\begin{array}{l}90.73 \\
\pm 7.80\end{array}$ & $\begin{array}{c}4.44 \\
\pm 0.10\end{array}$ & $\begin{array}{c}1.11 \\
\pm 0.03\end{array}$ & $\begin{array}{c}3.12 \\
\pm 0.13\end{array}$ & $\begin{array}{c}0.53 \\
\pm 0.02\end{array}$ & $\begin{array}{l}14.93 \\
\pm 0.75\end{array}$ & $\begin{array}{l}154.7^{\mathbf{b}} \\
\pm 2.21\end{array}$ & $\begin{array}{l}174.6 \\
\pm 3.58\end{array}$ & $\begin{array}{l}55.73^{\mathbf{b}} \\
\pm 1.90\end{array}$ & $\begin{array}{l}63.33^{\mathbf{b}} \\
\pm 2.33\end{array}$ \\
\hline Leaves of fruits & $\begin{array}{c}190.9 \\
\pm 22.54\end{array}$ & $\begin{array}{l}87.40 \\
\pm 6.87\end{array}$ & $\begin{array}{c}4.45 \\
\pm 0.08\end{array}$ & $\begin{array}{c}1.11 \\
\pm 0.04\end{array}$ & $\begin{array}{c}3.08 \\
\pm 0.09\end{array}$ & $\begin{array}{c}0.52 \\
\pm 0.02\end{array}$ & $\begin{array}{l}15.60 \\
\pm 0.94\end{array}$ & $\begin{array}{l}170.9^{\mathrm{a}} \\
\pm 4.63\end{array}$ & $\begin{array}{l}177.6 \\
\pm 2.47\end{array}$ & $\begin{array}{l}61.60^{\mathrm{a}} \\
\pm 1.50\end{array}$ & $\begin{array}{l}73.73^{\mathrm{a}} \\
\pm 4.37\end{array}$ \\
\hline \multicolumn{12}{|c|}{ Replacement rate $(\%)$} \\
\hline 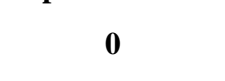 & $\begin{array}{c}169.2 \\
\pm 27.68\end{array}$ & $\begin{array}{l}86.00 \\
\pm 9.84\end{array}$ & $\begin{array}{l}4.13^{\mathbf{b}} \\
\pm 0.06\end{array}$ & $\begin{array}{l}1.22^{\mathrm{a}} \\
\pm 0.02\end{array}$ & $\begin{array}{l}2.70^{\mathbf{b}} \\
\pm 0.05\end{array}$ & $\begin{array}{c}0.55 \\
\pm 0.02\end{array}$ & $\begin{array}{l}14.80 \\
\pm 1.06\end{array}$ & $\begin{array}{l}158.0 \\
\pm 2.08\end{array}$ & $\begin{array}{l}174.0 \\
\pm 3.40\end{array}$ & $\begin{array}{l}61.60 \\
\pm 2.22\end{array}$ & $\begin{array}{l}61.60^{\mathbf{b}} \\
\pm 3.80\end{array}$ \\
\hline 25 & $\begin{array}{c}196.2 \\
\pm 28.14\end{array}$ & $\begin{array}{l}83.30 \\
\pm 8.06\end{array}$ & $\begin{array}{l}4.61^{\mathrm{a}} \\
\pm 0.07\end{array}$ & $\begin{array}{l}1.06^{\mathbf{b}} \\
\pm 0.05\end{array}$ & $\begin{array}{l}3.36^{\mathrm{a}} \\
\pm 0.11\end{array}$ & $\begin{array}{c}0.52 \\
\pm 0.03\end{array}$ & $\begin{array}{l}15.00 \\
\pm 0.86\end{array}$ & $\begin{array}{l}166.3 \\
\pm 7.28\end{array}$ & $\begin{array}{l}173.4 \\
\pm 4.57\end{array}$ & $\begin{array}{l}56.60 \\
\pm 2.43\end{array}$ & $\begin{array}{l}74.00^{\mathrm{a}} \\
\pm 5.55\end{array}$ \\
\hline 50 & $\begin{array}{c}201.5 \\
\pm 30.00\end{array}$ & $\begin{array}{l}97.90 \\
\pm 8.81\end{array}$ & $\begin{array}{l}4.58^{\mathrm{a}} \\
\pm 0.12\end{array}$ & $\begin{array}{l}1.04^{\mathbf{b}} \\
\pm 0.02\end{array}$ & $\begin{array}{l}3.24^{\mathrm{a}} \\
\pm 0.12\end{array}$ & $\begin{array}{c}0.50 \\
\pm 0.03\end{array}$ & $\begin{array}{l}16.00 \\
\pm 1.19\end{array}$ & $\begin{array}{l}164.0 \\
\pm 4.68\end{array}$ & $\begin{array}{l}180.9 \\
\pm 2.93\end{array}$ & $\begin{array}{l}57.80 \\
\pm 2.05\end{array}$ & $\begin{array}{c} \pm 70.00^{\mathbf{a b}} \\
\pm 3.49\end{array}$ \\
\hline \multicolumn{12}{|l|}{ Interactions } \\
\hline Skin * 0\% & $\begin{array}{c}169.2 \\
\pm 41.52\end{array}$ & $\begin{array}{c}86.00 \\
\pm 14.76\end{array}$ & $\begin{array}{c}4.13 \\
\pm 0.08\end{array}$ & $\begin{array}{c}1.22 \\
\pm 0.04\end{array}$ & $\begin{array}{c}2.70 \\
\pm 0.07\end{array}$ & $\begin{array}{c}0.55 \\
\pm 0.03\end{array}$ & $\begin{array}{l}14.80 \\
\pm 1.59\end{array}$ & $\begin{array}{l}158.0 \\
\pm 3.11\end{array}$ & $\begin{array}{l}174.0 \\
\pm 5.10\end{array}$ & $\begin{array}{l}61.60 \\
\pm 3.33\end{array}$ & $\begin{array}{l}61.60 \\
\pm 5.70\end{array}$ \\
\hline Skin $* 25 \%$ & $\begin{array}{c}236.0 \\
\pm 44.87\end{array}$ & $\begin{array}{c}83.40 \\
\pm 15.38\end{array}$ & $\begin{array}{c}4.73 \\
\pm 0.12\end{array}$ & $\begin{array}{c}1.04 \\
\pm 0.05\end{array}$ & $\begin{array}{c}3.52 \\
\pm 0.17\end{array}$ & $\begin{array}{c}0.52 \\
\pm 0.04\end{array}$ & $\begin{array}{l}14.60 \\
\pm 0.75\end{array}$ & $\begin{array}{l}151.4 \\
\pm 3.06\end{array}$ & $\begin{array}{l}168.0 \\
\pm 8.00\end{array}$ & $\begin{array}{l}52.60 \\
\pm 2.69\end{array}$ & $\begin{array}{l}63.20 \\
\pm 2.69\end{array}$ \\
\hline Skin $* \mathbf{5 0 \%}$ & $\begin{array}{r}156.0 \\
\pm 36.96\end{array}$ & $\begin{array}{r}102.80 \\
\pm 11.33\end{array}$ & $\begin{array}{c}4.45 \\
\pm 0.20\end{array}$ & $\begin{array}{c}1.07 \\
\pm 0.04\end{array}$ & $\begin{array}{c}3.14 \\
\pm 0.23\end{array}$ & $\begin{array}{c}0.51 \\
\pm 0.03\end{array}$ & $\begin{array}{r}15.40 \\
\pm 1.63\end{array}$ & $\begin{array}{r}154.6 \\
\pm 5.19\end{array}$ & $\begin{array}{l}181.8 \\
\pm 4.55\end{array}$ & $\begin{array}{l}53.00 \\
\pm 2.57\end{array}$ & $\begin{array}{l}65.20 \\
\pm 3.93\end{array}$ \\
\hline Leaves * $0 \%$ & $\begin{array}{c}169.2 \\
\pm 41.52\end{array}$ & $\begin{array}{r}86.00 \\
\pm 14.76\end{array}$ & $\begin{array}{c}4.13 \\
\pm 0.08\end{array}$ & $\begin{array}{c}1.22 \\
\pm 0.04\end{array}$ & $\begin{array}{c}2.70 \\
\pm 0.07\end{array}$ & $\begin{array}{c}0.55 \\
\pm 0.03\end{array}$ & $\begin{array}{r}14.80 \\
\pm 1.59\end{array}$ & $\begin{array}{l}158.0 \\
\pm 3.11\end{array}$ & $\begin{array}{r}174.0 \\
\pm 5.10\end{array}$ & $\begin{array}{r}61.60 \\
\pm 3.33\end{array}$ & $\begin{array}{r}61.60 \\
\pm 5.70\end{array}$ \\
\hline Leaves * $25 \%$ & $\begin{array}{c}156.4 \\
\pm 27.52\end{array}$ & $\begin{array}{l}83.20 \\
\pm 7.47\end{array}$ & $\begin{array}{c}4.49 \\
\pm 0.05\end{array}$ & $\begin{array}{c}1.09 \\
\pm 0.09\end{array}$ & $\begin{array}{c}3.20 \\
\pm 0.13\end{array}$ & $\begin{array}{c}0.51 \\
\pm 0.03\end{array}$ & $\begin{array}{r}15.40 \\
\pm 1.63\end{array}$ & $\begin{array}{c}181.2 \\
\pm 10.86\end{array}$ & $\begin{array}{r}178.8 \\
\pm 3.93\end{array}$ & $\begin{array}{r}60.60 \\
\pm 3.37\end{array}$ & $\begin{array}{l}84.80 \\
\pm 8.53\end{array}$ \\
\hline Leaves $* \mathbf{5 0 \%}$ & $\begin{array}{c}247.0 \\
\pm 40.61\end{array}$ & $\begin{array}{c}93.00 \\
\pm 14.46\end{array}$ & $\begin{array}{c}4.72 \\
\pm 0.11\end{array}$ & $\begin{array}{c}1.01 \\
\pm 0.02\end{array}$ & $\begin{array}{c}3.34 \\
\pm 0.09\end{array}$ & $\begin{array}{c}0.49 \\
\pm 0.06\end{array}$ & $\begin{array}{l}16.60 \\
\pm 1.89\end{array}$ & $\begin{array}{l}173.4 \\
\pm 5.25\end{array}$ & $\begin{array}{l}180.0 \\
\pm 4.18\end{array}$ & $\begin{array}{l}62.60 \\
\pm 0.93\end{array}$ & $\begin{array}{l}74.80 \\
\pm 5.28\end{array}$ \\
\hline P value & 0.1153 & 0.9157 & 0.1143 & 0.5917 & 0.1867 & 0.9725 & 0.9260 & 0.0507 & 0.4519 & 0.2138 & 0.1765 \\
\hline
\end{tabular}

a-b: Means superscripted with different letters in the same column differ significantly at $\mathrm{P} \leq 0.05$, AST: aspartate aminotransferase, ALT: alanine aminotransferase, GL: globulin, TP: total protein, TCH: total cholesterol, TG: triglycerides, HDL: high density lipoprotein, LDL: low density lipoprotein.

\section{DISCUSSION}

Results in the present study detected that the seriously affected of different nutritional treatments on hematological and biochemical parameters, which confirmed the potentially relationship between feeding systems and physiological responses not only in fish, but also in all farm or experimental animals. In this respect, Hussein et al. (2001) found that canola meal increased significantly $\mathrm{Hb}$, serum total protein, globulin, triglycerides, AST, ALT, and thyroid hormones levels. Dietary yeast strains increased $\mathrm{Hb}$, serum total protein, globulin, triglycerides, glucose, AST, ALT, and thyroid hormones levels. They gave the following ranges: $\mathrm{Hb}$ (6.4-9.3 g/dL), PCV (22.0-25.3\%), serum total protein (4.83-10.5 g/dL), albumin (1.74-2.64 g/dL), globulin (2.57-8.68 g/dL), triglycerides $(248-375 \mathrm{mg} / \mathrm{dL})$, glucose (93.5-128 mg/dL), total cholesterol (146-165 mg/dL), AST (38.8-66.4 u/L), and ALT (22.5-37.9 u/L). El-Ebiary and Zaki (2003) registered increases in the $\mathrm{Hb}$ (6.36-8.39 $\mathrm{g} / \mathrm{dL})$, PCV (23.1-25.0 \%), serum total protein (4.85-9.46 g/dL), albumin (2.26-264 $\mathrm{g} / \mathrm{dL})$, globulin (2.59-6.85 g/dL), glucose $(92.5-148 \mathrm{mg} / \mathrm{dL})$ and triglycerides' (255$356 \mathrm{mg} / \mathrm{dL}$ ) concentrations, but decrease of the total cholesterol level (156-143 $\mathrm{mg} / \mathrm{dL}$ ) by increasing the level of active yeast in the Nile tilapia diets. Red tilapia fed sesame hulls by-product reflected lower plasma glucose (78.6-67.2 mg/dL) and total protein concentrations (5.10 vs. $2.69 \mathrm{~g} / \mathrm{dL})$ and ALT activity (39.5-10.9 u/dL) comparing with the control (Abd Elmonem et al., 2004). Inversely, El-Houssiny et al. (1999) reported no significant $(\mathrm{P} \geq 0.05)$ differences in non-specific immunity 
factor (serum proteins) nor humoral immune response in Nile tilapia fed different protein levels $(20,25$, and $30 \%$ ), feeding levels ( 3 and $5 \%$ ), and feeding frequency ( 2 and 3 times) for 170 days. They gave the following ranges for serum total protein 2.51-2.91 g/dL, albumin 0.99-1.17 g/dL, globulin 1.48-1.83 g/dL, and albumin/ globulin 0.57-0.86.

Ayyat et al. (2004) fed Nile tilapia different levels of fish meal and zinc. They found that serum total protein $(5.00 / 5.69 \mathrm{~g} / \mathrm{dL})$, albumin $(2.90 / 3.65 \mathrm{~g} / \mathrm{dL})$, and AST $(30.4 / 36.6 \mathrm{u} / \mathrm{L})$ values significantly increased with increasing fish meal level in the fish diet, while urea-N (3.83/3.65 mg/dL) and ALT (16.2/12.8 u/L) values significantly decreased. Also, serum total protein $(4.60 / 5.84 \mathrm{~g} / \mathrm{dL})$, albumin $(2.68 / 3.39 \mathrm{~g} / \mathrm{dL})$, and creatinine $(0.90 / 1.02 \mathrm{mg} / \mathrm{dL})$ concentrations and AST activity $(29.6 / 37.2 \mathrm{u} / \mathrm{L})$ significantly increased with increasing $\mathrm{Zn}$ level in the fish diet, while ALT activity significantly decreased. Moreover, El-Dakar et al. (2004) found that dietary inclusion of graded levels of fennel seeds meal as a feed additive significantly affected some serum biochemical parameters, particularly total lipids (3.33-5.51 $\mathrm{g} / \mathrm{dL})$ and AST $(72-116 \mathrm{u} / \mathrm{dL})$ but did not affect glucose $(34.3-59.6 \mathrm{mg} / \mathrm{dL})$, total protein (5.03-5.43), and ALT (34-45 u/dL). Abdel-Tawwab et al. (2005) represented that Nile tilapia reflected slightly lower $\mathrm{Hb}$ and PCV values under crowding-stress condition but there was no significant on them due to dietary CP level. They added that RBCs count and glucose concentration in chronically stressed fish were elevated but plasma cortisol value was reduced particularly with increasing the dietary protein level. However, both transaminases (AST \& ALT) were not affected. That means that Nile tilapia may quickly adapted to high rearing density by enhancing feed quality, especially protein level in the diet to prevent the deleterious effect in fish farm. The recorded the following ranges: RBCs 1.26-1.61 $\times 10^{6} \mu / \mathrm{L}, \mathrm{Hb} 6.06-8.40$ $\mathrm{g} / \mathrm{dL}$, PCV 13.8-16.5\%, glucose 64.9-128 mg / dL, protein 2.07-3.45 g / dL, ALT 6.08-8.75 u / L and AST 17.4-65.9 u / L.

Several food additives and immunostimulants can enhance different innate factors. There is limited data available about the ontogenic development of the innate immunological system in fish (Magnadóttir, 2006). In this regard, Osman et al. (2010) registered that feeding with probiotics improved the $\mathrm{RBC}_{\mathrm{s}}$ count $\left(1.159 \times 10^{6}\right.$ vrs. $\left.0.999 \times 10^{6} / \mu \mathrm{L}\right), \mathrm{Hb}$ concentration $(5.42$ vrs. $4.77 \mathrm{~g} / \mathrm{dL})$, phagocyte activity, A/G ratio, and serum total protein level of Nile tilapia. Also, Nile tilapia fed spirulina (Arthrospira fusiformis) supplementation significantly decreased plasma AST (108 vrs. $115 \mathrm{u} / \mathrm{dL})$ and ALT (41 vrs $46 \mathrm{u} / \mathrm{dL})$ activity, but significantly increased plasma glucose $(63.87$ vrs. $58.11 \mathrm{mg} / \mathrm{dL})$, total protein $(7.58$ vrs $5.10 \mathrm{~g} / \mathrm{dL})$, and total lipid (5.28 vrs $4.23 \mathrm{~g} / \mathrm{dL}$ ) (Belal et al., 2012). Shortage of food supply and animal feedstuff gape forced the nutritionists to find out and evaluate novel or unconventional food resources such as corn gluten meal (Metwalli, 2013) and insect (black solider fly) larvae to replace the expensive fish meal in aquafeed (Makker, 2015). Al-Ashrm (2017) mentioned among the properties of an ideal narcotic agent, it must be not toxic to fish and human being, do not affect fish behavior or its physiological functions, and to be cheap. He added that clove oil (extracted from the plant Eugeaia caryaphyllata) is a local product, safe, effective, and cheap narcotic agent well recommended for fish narcosis.

Moazenzadeh et al. (2017) investigated the effect of dietary $\mathrm{Zn}$ levels $(0,5,10$, 20 , and $40 \mathrm{mg} \mathrm{Zn} / \mathrm{kg}$ ) on hematological parameters of juvenile Siberian sturgeon (Acipenser baerii, Brandt 1869). Erythrocyte number, Hb concentration, and Hct were increased with raising dietary $\mathrm{Zn}$ level $(\mathrm{P} \leq 0.05)$, whereas a regular trend was not observed in blood indices, including $\mathrm{MCV}, \mathrm{MCH}$, and $\mathrm{MCHC}$. A significant 
increase was also found in WBCs number followed by their differential counts, except for eosinophil percentage. In spite of well correlation with dietary $\mathrm{Zn}$ level, no break point was observed to estimate the juvenile Siberian sturgeon requirement based on RBCs numbers. More recently, Hassaan et al. (2018) fed Nile tilapia, Oreochromis niloticus (average initial weight, $5.91 \pm 0.04 \mathrm{~g}$ ) isonitrogenous and isolipidic diets for 84 days. The diets contained four levels of yeast extract rich in nucleotides and $\beta$-glucan: 0 (control), 5,10 and $15 \mathrm{~g} / \mathrm{kg}$ diet. No significant $(\mathrm{P}>0.05)$ differences were found in $\mathrm{Hct}, \mathrm{Hb}$ or total protein contents among the treatment groups. Blood sample profiles showed an increase in WBCs and RBCs of fish fed 15 $\mathrm{g} / \mathrm{kg}$ yeast extract in comparison with the other treatments. Fish fed the diets supplemented with 10 and $15 \mathrm{~g} / \mathrm{kg}$ yeast extract had significantly higher albumin and globulin levels than the control group, whereas decreased levels of cholesterol and triglycerides, AST and ALT, which were noted in fish, fed the diet supplemented with $15 \mathrm{~g} / \mathrm{kg}$ yeast extract.

\section{CONCLUSION}

Based on the previous data it could be concluded that the possible relationship between different nutritional treatments and fish physiological status. Additionally, the interpretation of blood data of fish must be done on light of the specific individual experiment condition because of absence of referenced ranges for fish haematological and biochemical measurements of different fish species.

\section{REFERENCES}

Abd Elmonem, A. I.; Shalaby, S. M. M.; El-Dakar, A. Y. and Sadrak, O. W. (2004). Nutritional evaluation of sesame hulls by-product as a non-conventional feedstuff in diets of red tilapia, Oreochromis niloticus $\times$ Oreochromis mosambicus. Alex. J. Agric. Res., 49 (2): 1 - 13.

Abdel-Tawwab, M.; Mousa, M.A.A., Sharaf, S.M. and Ahmed, M.H. (2005). Effect of crowding stress on some physiological functions of Nile tilapia, Oreochromis niloticus (L.) at different dietary protein levels. International Journal of Zoological Research, 1 (1): 41-47.

Al-Ashrm, A.M.M. (2017). Narcosis and fish health (clove oil for fish narcosis). Fish \& Feed, 42: 16-18.

Aly, S.M., Ahmed, Y.A. Ghareeb, A.A. and Mohamed, M.F. (2008). Studies on Bacillus subtilis and Lactobacillus acidophilus, as potential probiotics, on the immune response and resistance of tilapia nilotica (Oreochromis niloticus) to challenge infections. Fish and Shellfish Immunol., 25: 128 - 136.

Ayyat, S.M., Abbas, F.S., Sharaf, S.M. and El-Marakby, H.I. (2004). Growth performance, feed utilization and blood components of Nile tilapia Oreochromis niloticus as affected by dietary protein source and zinc supplementation. J. Egypt. Acad. Soc. Environ. Develop., (B-Aquaculture), 5 (1): $1-15$.

Belal, E.B.; Khalafalla, M.M.E. and El-Hais, A.M.A. (2012). Use of spirulina (Arthrospira fusiformis) for promoting growth of Nile tilapia fingerlings. African Journal of Microbiology research, 6 (35): 6423-6431.

Decie, S. I. V. and Lewis, S. M. (2006). Practical Hematology. $10^{\text {th }}$ Ed., Churchill Livingstone, London. ISBN: 13: 978- 443, PP: 736.

Duncan, D. (1955). Multiple range and multiple F-tests. Biometrics, 11: 1-42. 
El-Dakar, A.Y.; Hassanien, G.D.I., Gad, S.S. and Sakr, S.E. (2004). Medical and aromatic plants as feeding attractants for fish. 1- Effect of dried marjoram leaves on performance of hybrid tilapia, Oreochromis niloticus $\times$ Oreochromis auraus, fingerlings. J. Egypt. Acad. Soc. Environ. Develop., (B-Aquaculture), 5 (1): 67-83.

El-Ebiary E.H. and Zaki, M.A. (2003). Effect of supplementing active yeast to the diets on growth performance, nutrient utilization, whole body composition and blood constituents of mono-sex tilapia (Oreochromis niloticus). Egypt. J. Aquatic, Biol. \& Fish., 7 (1): 127-139.

El-Houssiny, O.M.; Shehata, T.M., Arafa, S.A. and Goda, A.M.A. (1999). Effect of dietary protein level and feeding regime on the humoral immune response of Nile tilapia (Oreochromis niloticus). Egypt. J. Aquat. Biol. \& Fish., 3 (1): 2538.

Hassaan, M.S.; Mahmoud, S.A., Sylwia Jarmolowicz, S., El-Haroun, E.R., Mohammady, E.Y. and Davies, S.J. (2018). Effects of dietary baker's yeast extract on the growth, blood indices and histology of Nile tilapia (Oreochromis niloticus L.) fingerlings. Aquaculture Nutrition, 24: 1709-1717.

Hussein, S.Y.; Soliman, I.A. and Abd El-Latif, A.H. (2001). Growth performance, blood constituents and thyroid hormones in Nile tilapia (Oreochromis niloticus) fed diets containing canola meal and supplemented with yeast strains. Assiut Vet. Med. J., 45 (90); 75-93.

Magnadóttir, B. (2006). Innate immunity of fish (overview). Fish \& Shellfish Immunology, 20: 137-151.

Makker, H. (2015). Insect larvae as fish meal replacement. Aquafeed (Feeding the farmed fish), November, pp. 25-26.

McGowan, M.W.; Artiss, J.D., Standbergh, D.R. and Zak, B.A. (1983). Peroxidasecoupled method for colorimetric determination of serum triglycerides. Clin. Chem., 29: 538.

Metwalli, A.A.A. (2013). Effect of partial and total substitution of fish meal with corn gluten meal on growth performance, nutrients utilization and some blood constituents of the Nile tilapia Oreochromis niloticus. Egypt. J. Quat. Biol. \& Fish., 17 (1): 91-100.

Moazenzadeh, K.; Islami, H.R., Zamini, A. and Soltani, M. (2017). Dietary zinc requirement of Siberian sturgeon (Acipenser baerii, Brandt 1869) juveniles based on the growth performance and blood parameters. Int. Aquat. Res., 9: 25-35.

Natt, M.P. and Herrick, C.A. (1952). A new blood diluent for counting erythrocytes and leucocytes of the chicken. Poultry Science, 31: 735-738.

Osman, H. A. M.; Ibrahim, T. B., Soliman, W. and Aboud, O. (2010). Improvement growth and immune status using a potential probiotic bacteria Micrococcus species among cultured Oreochromis niloticus. N. Y. Sci. J., 3 (10): 5-11.

Press, C. McL. and Evensen, O. (1999). The morphology of the immune system in teleost fishes. Fish \& Shellfish Immunology, 9: 309-318.

Sahan, A. and Duman, S. (2008). Effects of diet with $\beta-1,3 / 1,6$ glucan on some haematological parameters in common carp (Cyprinus carpio L., 1758) infected by ecto-parasites. Proc. of Middle East \& North Africa Conf. for Future of Animal Wealth, Cairo, Egypt, 16-18 Oct.

SAS (2006). SAS statistical guide for personal computer, SAS Institute Inc. Cary, NC. 
Tietz, N.W. (1986). Textbook of Clinical Chemistry. W.B. Saunders, Philadelphia, 1271.

Tietz, N.W. (1990). Clinical Guide to Laboratory Tests. $2^{\text {nd }}$ ed. Philadelphia.

Wotton, I.D. and Freeman, H. (1982). Microanalysis in Medical Biochemistry. Churchill, New York, USA.

\section{ARABIC SUMMARY}

\section{عوامل تؤثر على صورة دم الأسماك: أ) تأثير المعاملات الغذائية}

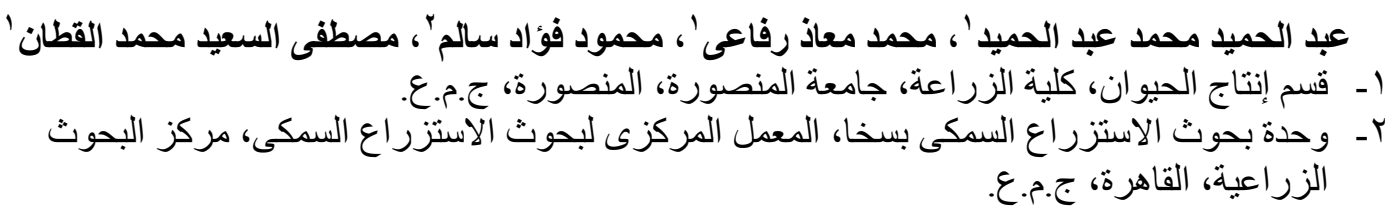

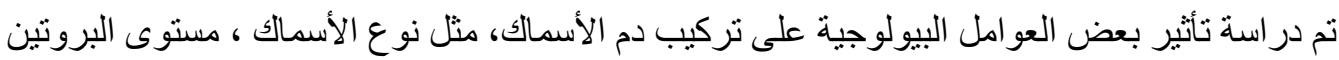

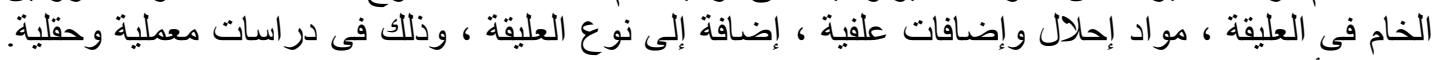

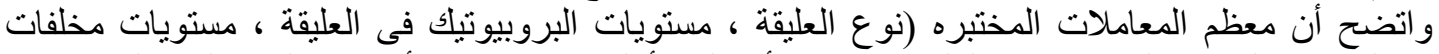

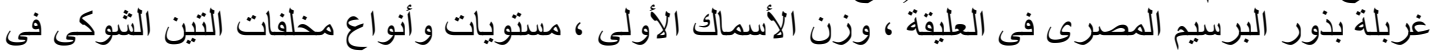

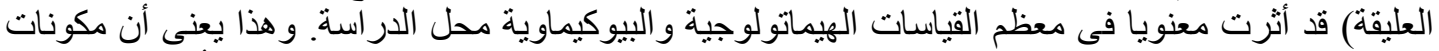

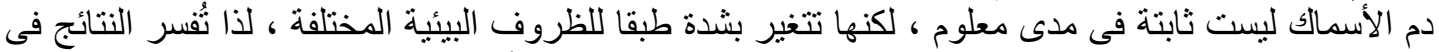

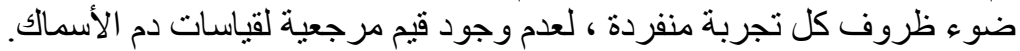

